\title{
A Large Reflective Schmidt Telescope for Antarctica
}

\author{
Will Saunders and Andrew McGrath \\ Anglo-Australian Observatory
}

\begin{abstract}
We present a simple design for a 16 metre, wide-field, fixed-axis, all-reflective, low cost $\mathrm{f} / 4$ Schmidt telescope to take advantage of the unique advantages of Antarctica as an Optical/IR site.
\end{abstract}

\section{Introduction}

Antarctica offers compelling advantages for Optical/IR astronomy, over and above the well-recognized gains of high transparency and low background.

1. It offers the unique possibility of single natural guide star AO-corrected observations over large fields-of-view. As well as allowing AO-corrected observations over much more of the sky, this opens up a whole new field of genuinely wide-field imaging at high spatial resolution.

2. The low temperatures remove most of the difficulties of IR telescope and camera optimization, allowing simpler and more general purpose designs.

3. ELT designs and costs are driven primarily by engineering considerations. The low wind speeds and seismic activity in Antarctica dramatically reduce the engineering difficulties.

In general, Schmidt telescopes offer the best optical quality and efficiency, over the largest possible fields of view, with the smallest possible number of elements. The Chinese 4m LAMOST design (www.lamost.org) shows how to build a big Schmidt - the axis is fixed, the corrector lens is replaced with a deformable segmented plain mirror, which corrects for spherical aberration as well as directing the light onto the fixed segmented spherical primary. The design is cheap and scale able. The speed is limited by the tilt on the corrector, which takes it away from the ideal, classical Schmidt position.

\section{Proposed design}

- LAMOST-style, horizontal axis, transit telescope. Can reach to equator and South Pole from Dome-C $\left(75^{\circ} \mathrm{S}\right)$.

- $16 \mathrm{~m}$ aperture, $\mathrm{f} / 4$, length $125 \mathrm{~m}$; Plate scale $300 \mu \mathrm{m} /{ }^{\prime \prime}$ to match $0.1^{\prime \prime}$ seeing.

- Segmented flat corrector mirror: steerable and deformable at $100 \mu \mathrm{m}$ level.

- Primary mirror fixed, spherical, segmented, phased.

- Partial vignetting at very large and very small elevations.

- Raised on 10-30m high ice pyramids (not shown) to get above ground-level turbulence.

- Shack-Hartmann module determines corrector Schmidt deformations; also corrects for tracking errors, windshake, mechanical sag; also provides adaptive wave- 


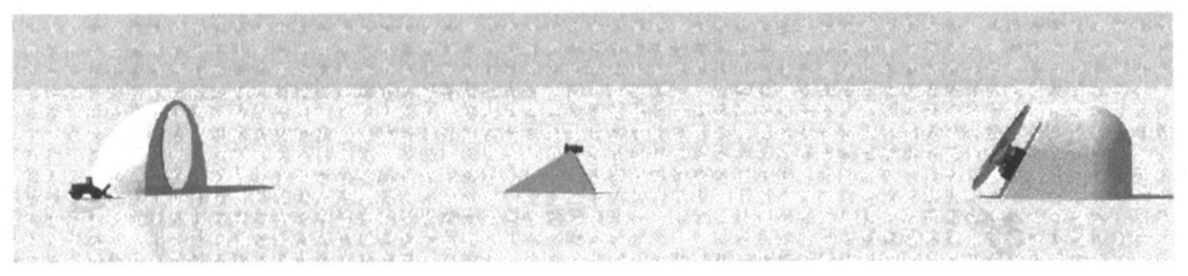

Figure 1. Proposed layout for $16 \mathrm{~m}$ Antarctic reflective Schmidt telescope

front correction at up to $100 \mathrm{~Hz}$ (fast enough for Antarctica).

- Focal stations: f/4 prime focus; and f/13, f/40 Cassegrain foci.

- IR uses baffles and Narcissus mirrors but no cold-stop. Increase in sky noise (over a perfect cold-stop) is few $\%$ at $K_{\text {dark }}(2.3 \mu \mathrm{m})$ and few tens of $\%$ at $L$ $(3.5 \mu \mathrm{m})$.

- Can easily guide to $\mathrm{R}=14.2$, can always find guide stars at Prime, and usually at Cassegrain $f / 13$, and over $\sim 10 \%$ of the sky at $f / 40$.

- Estimated cost at this stage (based on Hobby-Eberly, SALT, Keck) US\$100M$200 \mathrm{M}$ vs $\sim$ US $\$ 500 \mathrm{M}$ for other $25 \mathrm{~m}$-class ELT's.

\section{Focal stations and image quality}

- f/4 Prime Focus MOS: curved field plate: $300 \mu \mathrm{m} /{ }^{\prime \prime}, 0.2^{\prime \prime}$ fwhm over $3^{\circ}$

- $f / 4$ Prime Focus for imaging: flat-field, $0.05^{\prime \prime} /$ pixel, $0.06^{\prime \prime}$ fwhm over $0.5^{\circ}$

- $f / 13$ Cassegrain Focus: $0.015^{\prime \prime} /$ pixel, $0.025^{\prime \prime}$ fwhm over $4.5^{\prime}$

- $f / 40$ Cassegrain Focus: $0.005^{\prime \prime} /$ pixel, $0.015^{\prime \prime}$ fwhm over $1.5^{\prime}$

\section{Science Drivers}

At Prime Focus, the $A \Omega$ (Collecting Area $\times$ Solid Angle) for this design is 1-2 orders of magnitude larger than existing or proposed telescopes. For all thermal IR work, it would also be an order of magnitude more sensitive than other 25m-class ELT's, or NGST. Science drivers would include:

Wide-field Optical/NIR imaging at $0.1^{\prime \prime}$ fwhm: HDF-quality images over many square degrees. Complete evolution history of galaxies; LSS via lensing; stellar census in the Milky Way; stellar populations in nearby galaxies; in general, finding rare, faint objects.

Multiplexed fiber spectroscopy over $3^{\circ}$ fields: combination of aperture and FOV unparalleled for virtually all multi-object spectroscopy.

At Cassegrain, the image quality is as good as NGST or other proposed ELT's in the IR, but offers the prospect of AO corrected optical imaging, and either way do this over $1.5^{\prime}-5^{\prime}$ fields. Such a telescope could be expected to dominate 'deep fields' Optical/IR astronomy, high resolution optical astronomy, and all IR astronomy of faint objects. 